\title{
Survival outcomes and prognostic factors for ascending nasopharyngeal carcinoma (T4N0-1) receiving radiation therapy combined with chemotherapy: a population-based study from SEER database.
}

Fangzheng Wang ( $\nabla$ wangfz76@126.com )

Zhejiang Cancer Hospital Department of Radiation Oncology https://orcid.org/0000-0002-7823-1314

Jiang Chuner

Zhejiang Cancer Hospital

Piao Yongfeng

Zhejiang Cancer Hospital

Wang Lei

zhejiang cancer hospital

Yan Fengqin

zhejiang cancer hospital

Ye Zhimin

zhejiang cancer hospital

Fu Zhenfu

zhejiang cancer hospital

Jiang Haitao

zhejiang cancer hospital

Wang Yuezhen ( $\nabla$ wangyz@zjcc.org.cn )

zhejiang cancer hospital

Jiang Yangming

CAS

\section{Research}

Keywords: nasopharyngeal carcinoma, ascending, chemotherapy, radiation therapy, efficacy, prognosis

Posted Date: April 21st, 2020

DOI: https://doi.org/10.21203/rs.3.rs-22999/v1 
License: (c) (i) This work is licensed under a Creative Commons Attribution 4.0 International License. Read Full License 


\section{Abstract \\ Purpose}

This study aims to investigate survival outcomes and prognostic factors for upward nasopharyngeal carcinoma (NPC) patients receiving radiation therapy (RT) combined with chemotherapy (CT).

\section{Methods}

A total of 421 previously untreated, newly diagnosed T4N0-1 NPC patients, who were identified within the Surveillance, Epidemiology, and End Results (SEER) registry (years 2004-2015), were collected and retrospectively reviewed. All patients received treatment of RT and CT. Kaplan-Meier analysis was used to evaluate overall survival (OS) and cancer-specific survival (CSS). The differences in OS and CSS were compared using Log-rank test. The independent prognostic factors were established by using univariate and multivariate Cox proportional hazard models.

\section{Results}

With a median follow-up duration of 37 months (range: 3-154 months), the 5-year estimate OS and CSS rates were $59.3 \%$ and $63.7 \%$, respectively. NO and $\geq 65$ years were poor prognostic factors for OS and CSS. Moreover, histology and race were associated with OS and CSS. Univariate analysis indicated that $\geq 65$ years, NO, NHB and grade III were unfavorable independent prognosticators of OS and CSS. Multivariate analysis demonstrated that $\geq 65$ years, NO and NHB were correlated with poor OS and CSS.

\section{Conclusion}

Patients with stage T4N0-1 NPC receiving RT plus CT had favorable OS and CSS. Moreover, age, N stage and race were independent prognostic factors of OS and CSS.

\section{Introduction}

Nasopharyngeal cancer (NPC) is a unique malignant of head and neck, with the cute incidence of 15-50 cases per 100,000 annually in the endemic area such as southeast Asia, northern Africa and middle Europe ${ }^{[1]}$. The GLOBOCAN data ${ }^{[2]}$ in 2018 reported that there are 129097 patients with newly diagnosed NPC around the world. $47.7 \%$ of these patients occurred in China. Furthermore, the rate of loco-regionally advanced NPC (stage III or IV) accounted for about $70 \%{ }^{[3]}$. While $27 \%$ of newly diagnosed NPC patients presented with non-metastatic stage IV and the corresponding overall survival (OS) at 5 years was only about $65 \%{ }^{[4]}$. Although non-metastatic stage IV in the UICC 8th edition included both T4 and N3 ${ }^{[5]}$, there were three types of disease according to distinct clinical behavior and patterns of failure ${ }^{[6-8]}$. Three types 
of disease were described as follows: 1) ascending type: locally advanced disease with limited nodal spread (T4N0-1); 2) descending type: extensive metastatic lymph nodes and limited primary tumor (T1$2 \mathrm{~N} 3)$; 3) hybrid type: loco-regionally advanced disease (T3-4N2-3). For the patients with stage T4 NPC, local relapse was the main failure pattern, while distant metastasis was the predominant pattern of failure in stage N3 NPC patients. Mao et al reported that the local failure-free survival (LFFS) at 5 years was $76 \%$ for stage T4 patients and the distant metastasis-free survival (DMFS) at 5 years was $66 \%$ for stage N3 patients ${ }^{[4]}$. Huang et al investigated the survival and failure patterns of 3107 non-metastatic stage IV NPC patients and obtained the similar results ${ }^{[9]}$. Yao et al showed that compared with ascending types, descending types had poor survival and aggressive clinical course ${ }^{[8]}$. Given these situations, the treatment and survival outcomes were different among these three types of diseases. So, the aim of the current study was to investigate the survival outcomes and prognostic factors of the stage T4N0-1 NPC patient without distant metastasis.

\section{Materials And Methods}

\section{Database and patients' selection}

All histology-proven patients with NPC were identified in the Surveillance, Epidemiology, and End Results (SEER) database of the national Cancer Institute in the American. The SEER 18 database ${ }^{[10]}$ was obtained in the SEER program with SEEER ${ }^{*}$ Stat software, version 8.3.6 (www.seer.cancer.gov/seerstat). We selected the patients using the criteria as following: the patients were selected if age at diagnoses older than 20 years and diagnosed with NPC as the first malignancy from 2004 to 2015 . And we used the American Joint Committee on Cancer 6th or 7th edition staging to define the patients' stage. Stage N2-3 or distant metastases (M1) were exclusions. The therapeutic scheme for the NPC patients with T4N0-1 was the treatment of radiotherapy (RT) and/or chemotherapy (CT). Subjects received no treatment were excluded.

The flowchart of selective patients is shown in Fig. 1. A total of 1124 histology-confirmed NPC patients were registered in SEER database. A total of 421 NPC patients with T4N0-1 received RT plus CT were respectively reviewed. The study was exempt from our Institution Review Board because the information of all patients was publicly available. The information collected prospectively included patient demographics, histology, stage, therapeutic strategy, overall survival (OS) and cancer-specific survival (CSS).

\section{Statistical analyses}

All other data was analyzed by using IBM SPSS Statistical software, version 25.0. We used vital status and follow-up time from diagnosis date to calculated OS. And we used the cancer-specific death classification to compute CSS. The Kaplan-Meier method was used to generate survival curves and logrank tests were used compare the curves of different variables. We used a Cox regression model to conduct the univariate and multivariate in order to identify significant prognosticators. And we calculated 
hazard ratios (HRs) and 95\% confidence intervals (Cls) for each prognosticator. If a $P$ value $<0.05$, the differences were considered as statistically significant.

\section{Results}

\section{Patients' characteristics}

There were 421 patients with a mediate age of 55 years (range 20-86 years). Of these patients, the rate of male was $69.1 \%$, non-Hispanic White was $40.6 \%$, and $<65$ years was $79.6 \%$. All patients treated with RT and CT. Table 1 summarized the cohort characteristics.

Table 1 Basic characteristics of selected non-metastatic NPC patients with stage T4N0-1 from 2004 to 2015 in the SEER Database.

\begin{tabular}{cc}
\hline Characteristics & $\mathrm{N}(\%)$ \\
\hline Age & \\
Mediate (year) & 55 \\
Rang (year) & $20-86$ \\
$<65$ years & $335(79.6)$ \\
$\geq 65$ years & $86(20.4)$ \\
Gender & \\
Male & $291(69.1)$ \\
Female & $130(30.9)$ \\
Race & \\
Hispanic & $43(10.2)$ \\
NHAIAN & $10(2.4)$ \\
NHAPI & $160(38.0)$ \\
NHB & $37(8.8)$ \\
NHW & $171(40.6)$ \\
Grade & \\
Grade I & $11(2.6)$ \\
Grade II & $53(12.6)$ \\
Grade III & $133(31.6)$ \\
Grade IV & $110(26.1)$ \\
UNK & $114(27.1)$ \\
N stage* & \\
N0 & $193(45.8)$ \\
N1 & $228(54.2)$ \\
\hline &
\end{tabular}

Abbreviations: NHAIAN Non-Hispanic American Indian/Alaska Native; NHAPI Non-Hispanic Asia or Pacific Islander; NHB Non-Hispanic Black; NHW Non-Hispanic White; * The $6^{\text {th }}$ or th AJCC/UICC staging system

\section{Survival analyses}

The median survival time was 37 months (range, 3 to 154 months). The estimated OS and CSS rates at 5 years were $59.3 \%$ and $63.7 \%$, respectively (Fig. 2 ). The estimated rates OS at 5 years were $67.5 \%$ and $43.5 \%$ for patients with aged $<65$ years and $\geq 65$ years, respectively $(P<0.001$, Figure $3 \mathrm{~A})$. The estimated 5 -year CSS rates were $67.5 \%$ and $43.7 \%$ for patients with aged $<65$ years and $\geq 65$ years, respectively $(P<$ 
0.001 , Figure 3B). Interestingly, the estimated rates OS at 5 years were $49.7 \%$ and $66.3 \%$ for patients with grade N0 and N1, respectively ( $P<0.001$, Figure 3C), while the estimated 5 -year CSS rates were $54.1 \%$ and $70.5 \%$ for patients with grade N0 and N1, respectively ( $P=0.001$, Figure 3D).

\section{Identification of prognosticators}

Several potential prognosticators including age, gender, histology, race, and $\mathrm{N}$ stage. We used log-rank test to evaluate these prognostic factors, and the results showed that age, histology, and $\mathrm{N}$ stage were significant prognosticators for OS and CSS (Table 2). Interestingly, race was associated with OS and CSS.

Table 2 Results of univariable analysis of both OS and CSS in selected newly diagnosed NPC patients with T4N0-1 in the 18 SEER database. 


\begin{tabular}{|c|c|c|c|c|}
\hline Characteristics & 5-year OS & $\mathrm{P}$ & 5-year CSS & $\mathrm{P}$ \\
\hline \multicolumn{5}{|l|}{ Age } \\
\hline$<65$ years & 63.4 & $<0.001$ & 67.5 & $<0.001$ \\
\hline$\geq 65$ years & 38.9 & & 43.7 & \\
\hline \multicolumn{5}{|l|}{ Sex } \\
\hline Male & 59.3 & 0.543 & 63.5 & 0.720 \\
\hline Female & 56.4 & & 61.4 & \\
\hline \multicolumn{5}{|l|}{ Histology } \\
\hline Grade I & 29.8 & $<0.001$ & 34.1 & 0.012 \\
\hline Grade II & 42.3 & & 52.4 & \\
\hline Grade III & 57.8 & & 59.4 & \\
\hline Grade IV & 69.2 & & 71.3 & \\
\hline UNK & 59.3 & & 65.9 & \\
\hline \multicolumn{5}{|l|}{ Race } \\
\hline NHW & 58.7 & $<0.001$ & 60.2 & 0.005 \\
\hline NHB & 77.8 & & 77.8 & \\
\hline NHAIAN & 73.0 & & 76.2 & \\
\hline NHAPI & 37.7 & & 55.5 & \\
\hline Hispanic & 50.0 & & 53.2 & \\
\hline \multicolumn{5}{|l|}{$\mathrm{N}$ stage } \\
\hline No & 49.7 & $<0.001$ & 54.1 & 0.001 \\
\hline N1 & 66.3 & & 70.5 & \\
\hline
\end{tabular}

Abbreviations: NHAIAN Non-Hispanic American Indian/Alaska Native; NHAPI Non-Hispanic Asia or Pacific Islander; NHB Non-Hispanic Black; NHW Non-Hispanic White; NPC nasopharyngeal carcinoma; OS overall survival; CSS Cancer-specific survival; ${ }^{*}$ The $6^{\text {th }}$ or $7^{\text {th }}$ AJCC $/$ UICC staging system

Univariable Cox regression demonstrated that $<65$ years was associated with longer OS and CSS compared to $\geq 65$ years (OS HR=2.293, 95\% Cl: 1.673-3.14, P<0.001; CSS HR=2.34, 95\% Cl: 1.651-3.316, $\mathrm{P}<0.001$; Fig 4), while N1, compared to N0, provided the advantages of OS and CSS (OS HR $=0.599,95 \%$ Cl: $0.499-0.799, P=0.001$; Fig 4). Interestingly, grade III compared to grade I was associated with better OS 
and CSS (OS HR=2.2, 95\% Cl: 1.413-3.426, P<0.001; CSS HR=1.907, 95\% Cl: 1.132-3.214, $\mathrm{P}=0.015$; Fig 4). And, NHB compared to Hispanic was associated with unfavorable OS and CSS (OS HR=0.469, 95\% Cl: 0.333-0.659, P<0.001; CSS HR=0.492, 95\% Cl: 0.34-0.713, P<0.001; Fig 4).

Furthermore, multivariable Cox regression showed that $<65$ years was associated with longer OS and CSS compared to $\geq 65$ years (OS HR $=0.503,95 \%$ Cl: $0.361-0.702$, P<0.001; CSS HR=0.481, 95\% Cl: $0.332-$ $0.695, P<0.001$; Fig 5), while N1, compared to N0, provided the advantages of OS and CSS (OS HR=1.565, 95\% Cl: 1.163-2.104, P=0.003; Fig 5). Most interestingly, NHB compared to Hispanic was associated with unfavorable OS and CSS (OS HR=0.626, 95\% Cl: 0.433-0.904, P=0.012; CSS HR=0.628, 95\% Cl: 0.4200.938, P=0.023; Fig 5).

\section{Discussion}

The latest National Comprehensive Cancer Network (NCCN) guideline in 2020 ${ }^{[11]}$ recommends that chemoradiotherapy (CCRT) with induction chemotherapy (IC) or adjuvant chemotherapy (AC) is recommended as the first line treatment for locoregionally advanced NPC. The therapeutic schemes recommended by NCCN guideline were mainly used for the stage II-IV NPC patients without distant metastasis. While CCRT with or without AC was recommended for the treatment of stage IVA and IVB NPC patients by the European Society of Medical Oncology guideline ${ }^{[12]}$. Moreover, IC prior to CCRT was used to treat the NPC patients with tumor in close to important organs at risk. As stage IV NPC patients without distant metastasis is a special clinical entity, the survival outcomes was unsatisfactory although the combined strategy. In contrast to stage III NPC, stage IV disease had a worse prognostic factor. Due to different survival and prognosis of every type of disease, it is necessary to recognize each type of disease and to design individualized treatment option. Thus, the present study was to investigate the survival outcomes and prognostic factors of ascending NPC patients.

In the current study of stage T4N0-1 NPC patients without distant metastasis, we carefully identified the appropriate patients from the 18 SEER database and investigated the survival outcomes and prognosis. These findings indicated that the OS and CSS rates at 5 years were $59.3 \%$ and $63.7 \%$, respectively. On our univariate and multivariate analysis, $<65$ years was associated with longer OS and CSS compared to $\geq$ 65 years (OS HR $=0.503,95 \% \mathrm{Cl}: 0.361-0.702, \mathrm{P}<0.001$; CSS HR $=0.481,95 \% \mathrm{Cl}: 0.332-0.695, \mathrm{P}<$ 0.001 ), while N1, compared to NO, provided the advantages of OS and CSS (OS HR $=1.565,95 \% \mathrm{Cl}$ : 1.163-2.104, $P=0.003)$. Most interestingly, NHB compared to Hispanic was associated with unfavorable OS and CSS (OS HR $=0.626,95 \% \mathrm{Cl}: 0.433-0.904, \mathrm{P}=0.012 ;$ CSS HR $=0.628,95 \% \mathrm{Cl}: 0.420-0.938, \mathrm{P}=$ $0.023)$.

The stage T4N0-1 NPC patients had the lowest local control and relatively low distant metastasis. The 5 years local control rate was only $70-80 \%{ }^{[4]}$. Local relapse is an important factor affecting the efficacy of ascending NPC. If the patients experienced local relapse, the OS rate at 5 years was about $37-41 \%$, and $51 \%$ of these patients experienced radiation injury including nasopharyngeal necrosis and hemorrhage, which reduced the quality of life of the patients ${ }^{[13,14]}$. So, it is crucial to improve local 
control of these patients. The reasons of local relapse included clinical and biological factors. Clinical factor meant that insufficient RT dose of tumor resulted in local recurrence, while biological factors referred as resistance to RT for tumor cells. $\mathrm{Ng}$ and colleagues evaluated the effect of dosimetric inadequacy in target volumes on local control of NPC, the results indicated that if the volume below $66.5 \mathrm{~Gy}$ in gross tumor volume (GTV) was more than $3 \mathrm{cc}$, the 5 -year rate of local failure-free dropped to $54 \%{ }^{[15]}$. Due to the tumor in close to organ at risk (OAR), adaptive RT could be recommended to improve local control, but it needed randomized trial to validate therapeutic effect. However, hypoxia led to RT resistance ${ }^{[16]}$, while hyperbaric oxygen treatment ${ }^{[17]}$, red blood cell transfusion and erythropoietin delivery before or during the period of RT increased tumor oxygenation.

Although the ascending NPC belonged to locoregionally advanced NPC, it had different therapy option and survival patterns ${ }^{[6,7,18]}$. Thus, the optimal treatment strategy and survival outcomes of these patients need be investigated. Xiao et al performed the retrospective study of 148 T4 NPC patients treated with CCRT and the results indicated that the PFS and OS rates at 4 years were $46.9 \%$ and $75 \%$, respectively ${ }^{[19]}$. Chen et al demonstrated that for the T4 NPC patients receiving CCRT, the OS, DMFS, LRRFS and PFS rates at 4 years were $78.1 \%, 72.2 \%, 81.2 \%$ and $61.9 \%$, respectively ${ }^{[20]}$. Yao et al showed that IC + RT, compared to IC + CCRT or CCRT, was an encouraging treatment option for ascending NPC [21]. Moreover, Yao et al indicated that IC + RT provided similar survival and fewer complications for ascending NPC ${ }^{[22]}$. In addition, Zhang et al showed that CCRT combined nimotuzumab obtained a favorable local control and acceptable side events ${ }^{[23]}$.

Although SEER database provides a public available data to investigate this clinical problem, several limitations were observed in this study. Firstly, treatment information including RT dosing, CT regimen, delays in CT and therapy dates was not registered into SEER database, and we did not analyze the role of these factors. Secondly, therapy-related complications were not evaluated in this study due to lack of the information about RT- and CT-related side events. In addition, SEER records did not include the information about locoregional relapse, distant metastasis, so we failed to assess LRRFS and DMFS.

Although there are some limitations in the present study, it indicated that the 5-year OS and CSS rates in the ascending NPC patients were $59.3 \%$ and $63.7 \%$, respectively. On our univariate and multivariate analysis, $<65$ years was associated with longer OS and CSS compared to $\geq 65$ years, while N1, compared to NO, provided the advantages of OS and CSS. Most interestingly, NHB compared to Hispanic was associated with unfavorable OS and CSS. Thence, the prospective phase III trails needed to validate these gains.

\section{Conclusion}

The current study indicated that the 5-year OS and CSS rates in the stage T4N0-1 NPC patients without distant metastasis from the 18 SEER database were $59.3 \%$ and $63.7 \%$, respectively. Moreover, age, race and N stage were independent prognostic factors of OS and CSS for ascending NPC patients. It needs to conduct further multicenter prospective clinical trials to verify the ultimate benefits. 


\section{Abbreviations}

NPC=nasopharyngeal carcinoma;

UICC=Union for International Cancer Control

$\mathrm{RT}=$ radiotherapy

CT =chemotherapy

LFFS=local failure-free survival

DMFS=distant metastasis-free survival

SEER=Surveillance, Epidemiology and End Results

IBM SPSS=International Business Machines Statistical Product and Service Solutions

HRs=hazard ratios

Cls=confidence intervals

NHAIAN=Non-Hispanic American Indian/Alaska Native;

NHAPI=Non-Hispanic Asia or Pacific Islander;

NHB=Non-Hispanic Black;

NHW=Non-Hispanic White;

OS=overall survival;

CSS=Cancer-specific survival;

$\mathrm{NCCN}=$ National Comprehensive Cancer Network

CCR=chemoradiotherapy

IC=induction chemotherapy

$\mathrm{AC}=$ adjuvant chemotherapy

GTV=gross tumor volume

$\mathrm{OAR}=$ organ at risk

LRRFS=ocoergional relapse-free survival 


\section{Declarations}

Ethics approval and consent to participate: The study was exempt from our Institution Review Board because the information of all patients was publicly available.

Consent for publication: Not applicable.

Availability of data and materials: Not applicable

Conflicts of interest: The authors declare that they have no competing interests.

Funding: This study was supported by grants from the Medical and Health Science and Technology Program of Zhejiang Province (No.2020KY084, No.2019KY041, No. 2013KYB033, No. 2009B026, No. 2006A016, No. 2005B012, No. 2004B014), National Natural Science Foundation of China (No. 81502647).

\section{Author contributions:}

Conception and design: Fangzheng Wang, Yuezhen Wang, Zhenfu Fu, Yangming Jiang.

Acquisition of data: Chuner Jiang, Lei Wang, Haitao Jiang, Fengqin Yan, Zhimin Ye, Yongfeng Piao.

Data analysis and interpretation: Fangzheng Wang, Yangming Jiang.

Drafting the article and revising it critically for important intellectual content: Yongfeng Piao, Fangzheng Wang, Zhenfu Fu, Yangming Jiang.

Final approval of manuscript: All authors

Acknowledgements: None

\section{References}

1. Tang LL, Chen WQ, Xue WQ, et al. Global trends in incidence and mortality of nasopharyngeal carcinoma. Cancer Lett. 2016;374:22-30.

2. Bray F, Ferlay J, Soerjomataram I, et al. Global cancer statistics 2018: GLOBOCAN estimates of incidence and mortality worldwide for 36 cancers in 185 contries. CA Cancer J Clin. 2018;68:394424.

3. Mao YP, Xie FY, Liu LZ, et al. Re-evaluation of 6th edition of AJCC staging system for nasopharyngeal carcinoma and proposed improvement based on magnetic resonance imaging. Int J Radiat Oncol Biol Phys. 2009;73:1326-34. 
4. Au KH, Ngan RKC, Ng AWY, et al. Treatment outcomes of nasopharyngeal carcinoma in modern era after intensity modulated radiotherapy (IMRT) in Hong Kong: A report of 3328 patients (HKNPCSG 1301 study). Oral Oncol. 2018;77:16-21.

5. Amin MB, Edge S, Greene F. AJCC cancer staging manual. 8th ed. New York: Springer; 2017.

6. Xie ZG, Li ZQ. Natural development of nasopharyngeal carcinoma and clinical types of late stage cases. Tientsin Med J. 1963;1:129-31.

7. Li ZQ, Pan QC, Chen JJ. Clinical and experiment study of nasopharyngeal carcinoma. 1st ed. Guangzhou: Guangdong Science and Technology Publishing House; 1983.

8. Yao JJ, Qi ZY, Liu ZG, Jiang GM, Xu XW, Chen SY, et al. Clinical features and survival outcomes between ascending and descending types of nasopharyngeal carcinoma in the intensity-modulated radiotherapy era: a big-data intelligence platform-based analysis. Radiother Oncol. 2019;137:13744.

9. Huang CL, Guo R, Li JY, et al. Nasopharyngeal carcinoma treated with intensity-modulated radiotherapy: clinical outcomes and patterns of failure among subsets of 8th AJCC stage IVa. Eur Radiol. 2019.

10. Surveillance, Epidemiology, and End Results (SEER) Program (www.seer. cancer.gov) SEER*Stat Database: Incidence-SEER 18 Regs Custom Data (with additional treatment fields), Nov 2016 Sub (2004-2015 varying) - Linked to County Attributes-Total U.S., 1969-2016 Counties, National Cancer Institute, DCCPS, Surveillance Research Program, released April 2018, based on the November 2017 submission. Available at: https://seer. cancer.gov/data. Accessed July 22, 2018.

11. National Comprehensive Cancer Network. NCCN Clinical Practice Guide- lines Head and Neck Cancers. Version 1.2020. Available at: https://www. nccn.org/professionals/physician_gls/default.aspx\#head-and-neck. Accessed February 12, 2020.

12. Chan AT, Gregoire V, Lefebvre JL, et al. Nasopharyngeal cancer: EHNS-ESMO-ESTRO Clinical Practice Guidelines for diagnosis, treatment and follow-up. Ann Oncol. 2012;23(Suppl 7):vii83-85.

13. Tian $Y M$, Tian $Y H$, Zeng $L$, et al. Prognostic model for survival of local recurrent nasopharyngeal carcinoma with intensity- modulated radiotherapy. Br J Cancer. 2014;110:297-303.

14. Han F, Zhao C, Huang SM, et al. Long-term outcomes and prognostic factors of re-irradiation for locally recurrent nasopharyngeal carcinoma using intensity-modulated radiotherapy. Clin Oncol ( $R$ Coll Radiol). 2012;24:569-76.

15. Ng WT, Lee MC, Chang AT, et al. The impact of dosimetric inadequacy on treatment outcome of nasopharyngeal carcinoma with IMRT. Oral Oncol. 2014;50:506-12.

16. Overgaard J. Hypoxic modification of radiotherapy in squamous cell carcinoma of the head and neck-a systematic review and meta-analysis. Radiother Oncol. 2011;100:22-32.

17. Yoshimura $\mathrm{M}$, Itasaka $\mathrm{S}$, Harada $\mathrm{H}$, et al. Microenvironment and radiation therapy. Biomed Res Int. 2013; 2013: 685308.

18. Li ZQ, Xia YF, Liu Q, et al. Radiotherapy-related typing in 842 patients in canton with nasopharyngeal carcinoma. Int J Radiat Oncol Biol Phys. 2006;66:1011-6. 
19. Xiao C, Wang L, Jiao Y, et al. Long-term results of concurrent chemoradiotherapy for T3/T4 locally advanced nasopharyngeal carcinoma. Mol Clin Oncol. 2013;1:507-10.

20. Chen JL, Huang YS, Kuo SH, et al. Intensity-modulated radiation therapy for T4 nasopharyngeal carcinoma: treatment results and locoregional recurrence. Strahlenther Onkol. 2013;189:1001-8.

21. Yao JJ, Zhou GQ, Zhang F, et al. Neoadjuvant and concurrent chemotherapy have varied impacts on the prognosis of patients with the ascending and descending types of nasopharyngeal carcinoma treated with intensity-modulated radiotherapy. PloS ONE. 2016;11:e0161878.

22. Yao JJ, Yu XL, Zhang F, et al. Radiotherapy with neoadjuvant chemotherapy versus concurrent chemoradiotherapy for ascending-type nasopharyngeal carcinoma: a retrospective comparison of toxicity and prognosis. Chin J Cancer. 2017;36:26.

23. Zhang S, Huang XP, Zhou LY, et al. Efficacy of concurrent chemotherapy combined with nimotuzumab for low-risk T4 stage nasopharyngeal carcinoma: a pilot study. Medicine. 2018;97:e12503.

\section{Figures}




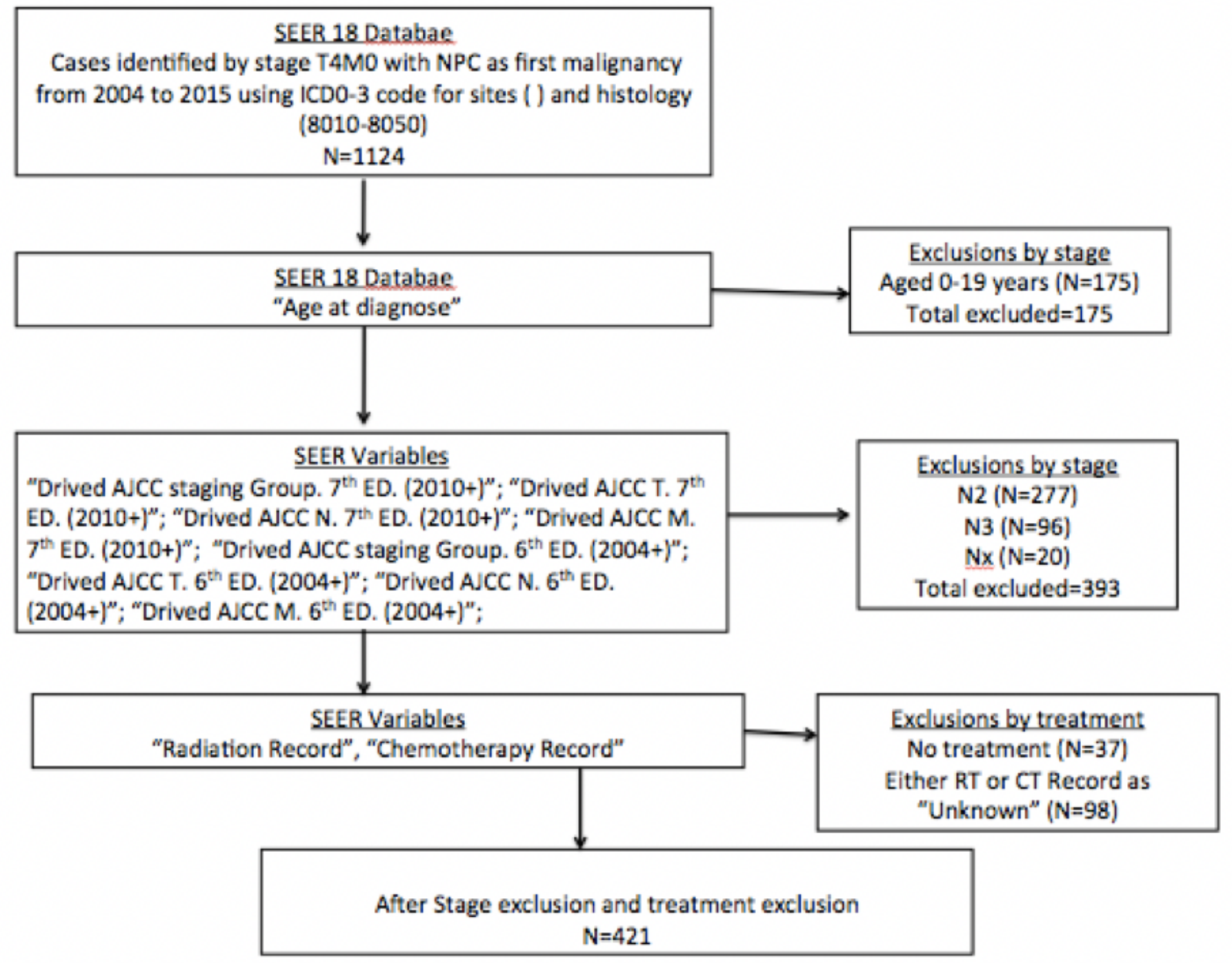

Figure 1

Flowchart of selective patients with aged $\geq 20$ years and nasopharyngeal carcinoma diagnosed from 2004 to 2015 in the SEER registry based on T4N0-1. NPC=nasopharyngeal carcinoma; ICD-

03=International Classification of Diseases for Oncology, 3rd Edition; AJCC=American Joint Committee on Cancer; SEER=Surveillance, Epidemiology, and End Results; 

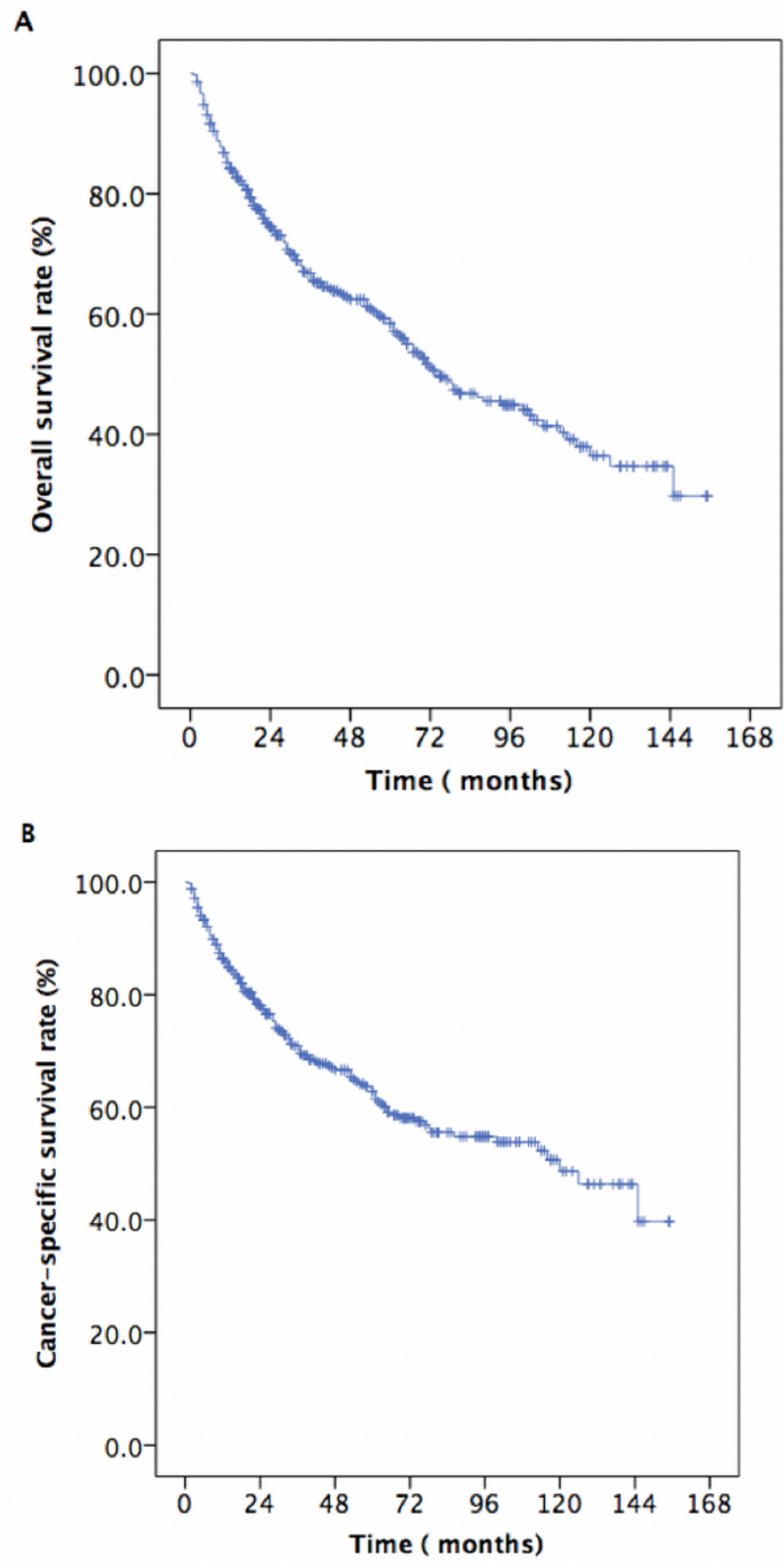

Figure 2

Kaplan-Meier estimates of the survival in newly diagnosed nasopharyngeal carcinoma patients with stage T4N0-1. (A) Overall survival; (B) Cancer-specific survival 

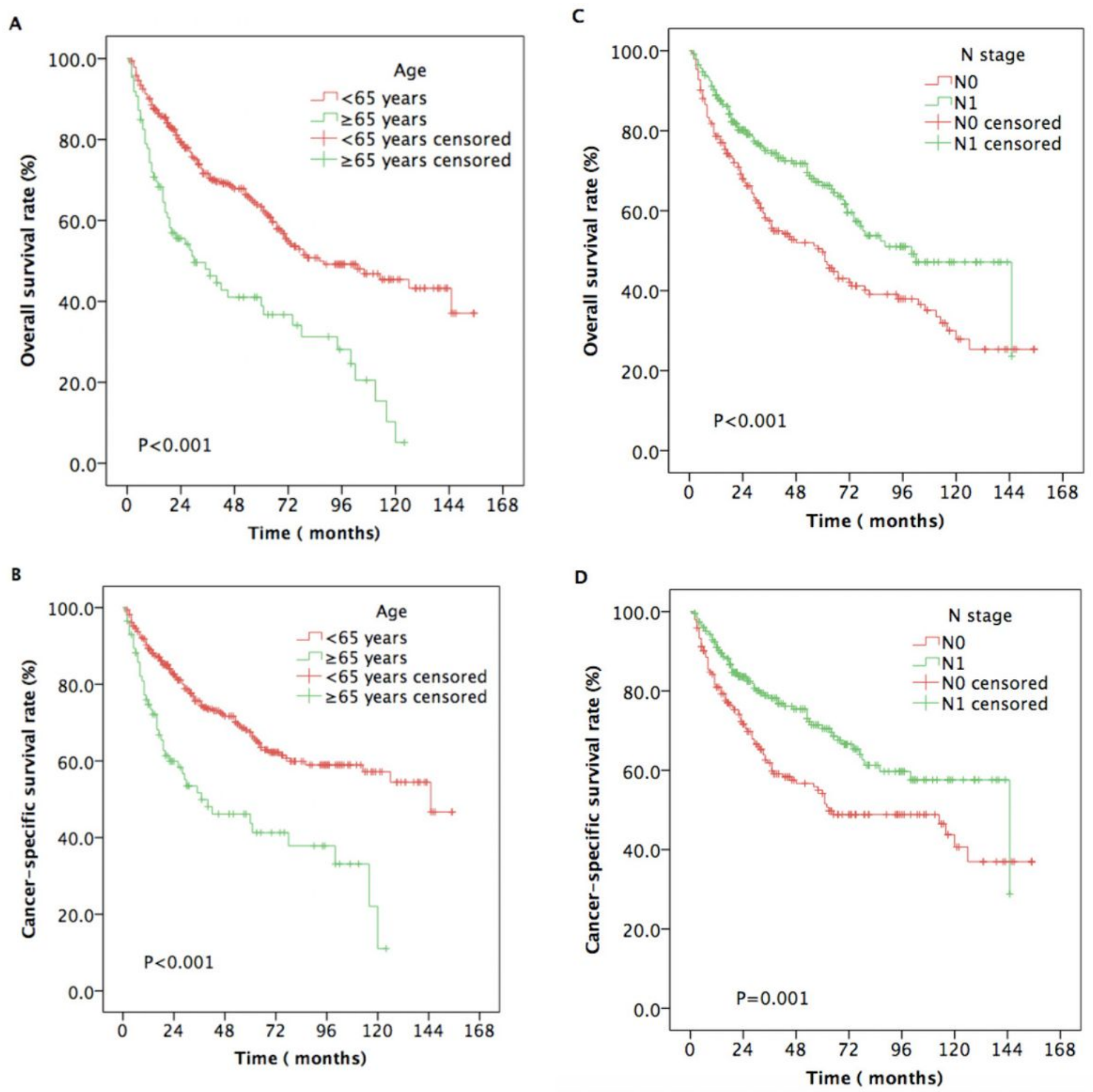

Figure 3

Kaplan-Meier estimates of the survival in newly diagnosed nasopharyngeal carcinoma patients with stage T4N0-1 for different variables. (A) Overall survival for age; (B) Cancer-specific survival for age; (C) Overall survival for $\mathrm{N}$ stage; (D) Cancer-specific survival for $\mathrm{N}$ stage; 
os

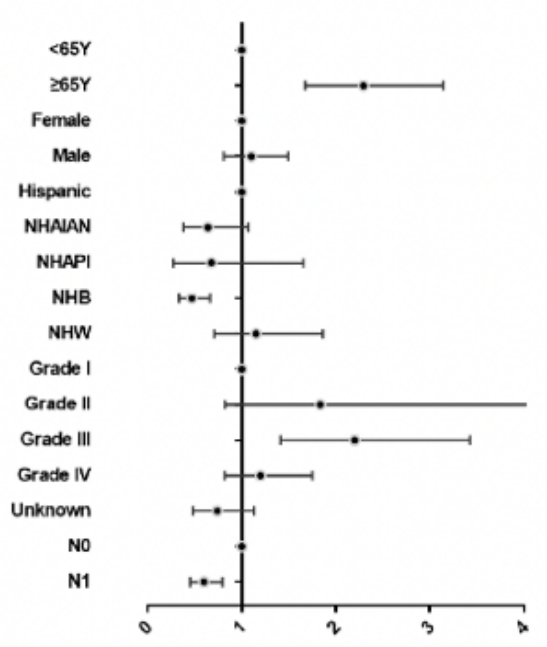

css

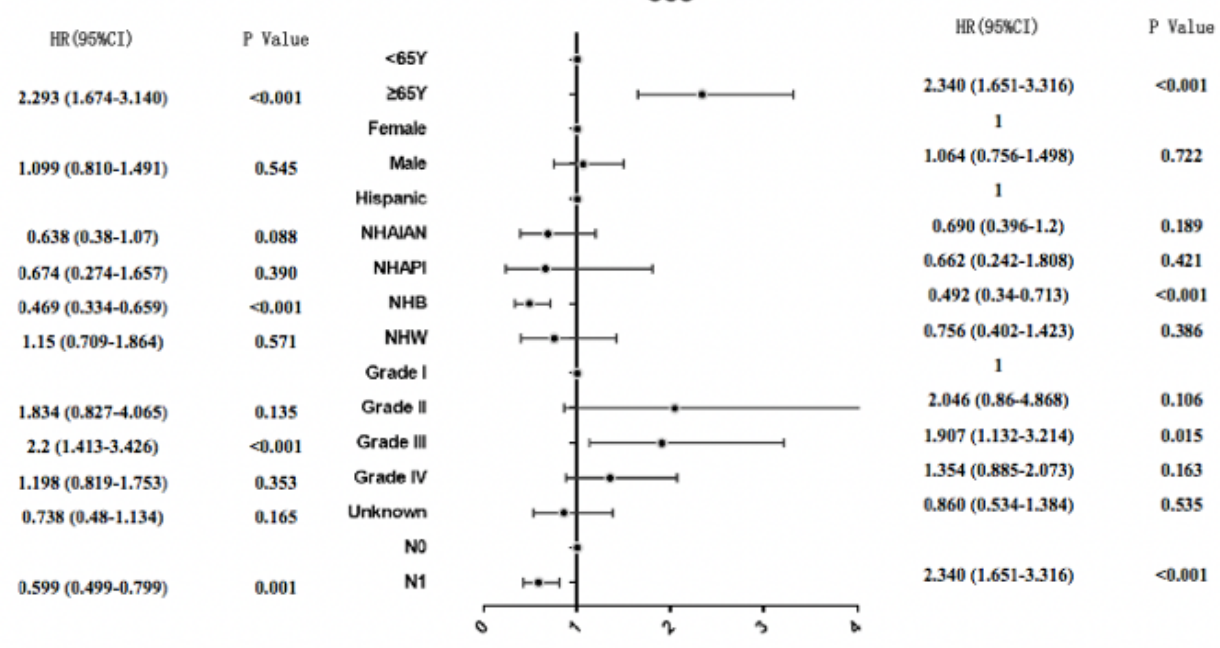

\section{Figure 4}

weighted univariable Cox proportional hazards regression analyses are shown for overall survival and cancer-specific survival

os

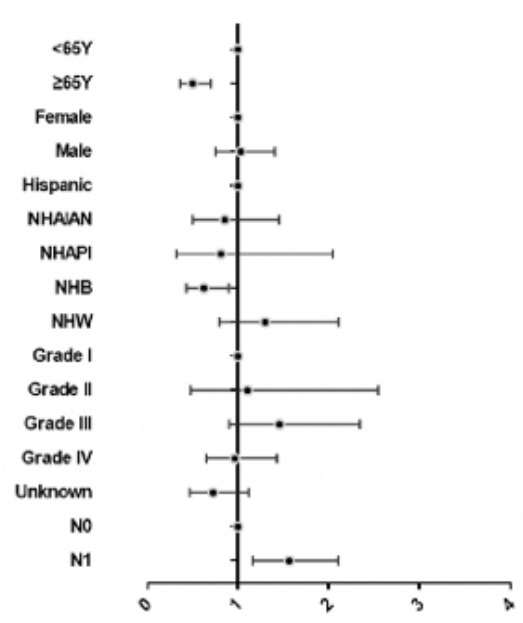

css

$\begin{array}{cc}\text { HR (954CI) } & \text { P Value } \\ 0.503(0.361-0.702) & 40.001 \\ & \\ 1.033(0.757-1.409) & 0.838 \\ & \\ 0.858(0.503-1.451) & 0.560 \\ 0.812(0.323-2.043) & 0.658 \\ 0.626(0.433-0.904) & 0.012 \\ 1.296(0.795-2.111) & 0.299 \\ & \\ 1.105(0.48-2.543) & 0.814 \\ 1.458(0.906-2.346) & 0.12 \\ 0.966(0.652-1.432) & 0.865 \\ 0.725(0.47-1.119) & 0.147 \\ & \\ 1.565(1.163-2.104) & 0.003 \\ \end{array}$

$\begin{array}{cc}\text { HR (95kCI) } & \text { P Value } \\ 0.481(0.332-0.695) & <0.001 \\ 1 & \\ 0.992(0.7-1.405) & 0.963 \\ 1 & \\ 0.895(0.507-1.579) & 0.702 \\ 0.798(0.285-2.239) & 0.669 \\ 0.628(0.420-0.938) & 0.023 \\ 0.847(0.448-1.600) & 0.609 \\ 1 & \\ 0.162(0.468-2.886) & 0.747 \\ 1.253(0.716-2.194) & 0.429 \\ 1.083(0.698-1.680) & 0.723 \\ 0.841(0.521-1.359) & 0.480 \\ & \\ 1.570(1.129-2.183) & 0.007\end{array}$

\section{Figure 5}

weighted multivariable Cox proportional hazards regression analyses are shown for overall survival and cancer-specific survival 